Original Research Article

\title{
Analysis of lead and arsenic in cosmetics and assessment of students awareness about cosmetic toxicity
}

\author{
Rehna Saidalavi ${ }^{1}$, Hashim A. ${ }^{2 *}$, Kishor Kumar B. ${ }^{2}$, Leena Pramod K. ${ }^{2}$, Prabhakar Adake
}

\begin{abstract}
${ }^{1}$ Department of Emergency Medicine, ${ }^{2}$ Department of

Forensic Medicine and Toxicology, ${ }^{3}$ Department of Pharmacology, Yenepoya Medical College, Yenepoya University, Karnataka, Mangalore, India
\end{abstract}

Received: 28 March 2017 Accepted: 27 April 2017

\section{*Correspondence to:}

Mr. Hashim A.,

Email: mhdhashim@gmail.com

Copyright: (C) the author(s), publisher and licensee Medip Academy. This is an openaccess article distributed under the terms of the Creative Commons Attribution NonCommercial License, which permits unrestricted noncommercial use, distribution, and reproduction in any medium, provided the original work is properly cited

\begin{abstract}
Background: Many people believe that all cosmetics are safe but there are number of studies proving that majority of the cosmetics contains heavy metals or other chemicals. In this study we selected two heavy metals arsenic and lead because as per the Indian scenario is concerned most of the Hindu and Muslim females uses lead/arsenic based cosmetics.

Methods: For the qualitative/quantitative estimation to detect heavy metals such as arsenic and lead in cosmetics, 20 different types of cosmetics were purchased from local market, labelled and analysed by qualitative test for heavy metals method and ICP-AES Analysis of cosmetics method.

Results: A questionnaire was framed out in English and analysis showed that students had an adverse effect while using cosmetic products. Preliminary analysis of some samples gave positive results and for confirmation these samples were sent to IIT Bombay for ICP-AES analysis. Some of the samples showed variable level of heavy metals such as lead and arsenic. Highest concentration of lead and arsenic was found in Surma followed by Sindoor, lip balm and Kajal.

Conclusions: Heavy metals are one of the major contaminant in our eco system. Apart from this a number of cosmetic products also contain the same. Chronic use of this kind of cosmetic products will leads heavy metal toxicity.
\end{abstract}

Keywords: Cosmetic toxicity, Cosmetic products, Heavy metals, Surma, Sindoor

\section{INTRODUCTION}

Cosmetics (colloquially known as makeup or make-up) are care substances used to enhance the appearance or odour of the human body. They are generally mixtures of chemical compounds, some being derived from natural sources, many being synthetic. Cosmetics ingredients come from a variety of sources but, unlike the ingredients of food, are often not considered by most consumers. Some of the cosmetic ingredients are made from crushed insects.

Cosmetics include skin-care creams, lotions, powders, perfumes, lipsticks, fingernail and toe nail polish, eye and facial makeup, towelettes, permanent waves, hair colours, hair sprays and gels, deodorants, shampoos and many other types of products.

People who use and work with cosmetics are in danger. Topical application of these is absorbed to some degree. Application of cosmetics over a period of time causes low-grade toxicity. Most have been scarcely tested, and only a few have had extensive toxicity testing. Allergic responses are very common with the use of cosmetic. Some of the ingredients in cosmetics and personal care products are toxic chemicals that may be hazardous to our health. Heavy metals like lead, arsenic and cadmium sound more like a chemical-laden concoction rather than ingredients found in popular cosmetics. The heavy metals found in the products tested are considered as unintentional contaminants or intentionally added to get 
desirable effect. These heavy metals are added to the cosmetics for colour, odour and increase surface adhesion or for biological response. ${ }^{1}$

Most of these heavy metals are highly poisonous to human body; The effects of poisons, which are absorbed through the skin, causes a lot of health problems, including hormone imbalance, cancer, neurological problems, memory loss, mood swings, reproductive and developmental disorders, kidney problems, headaches, dermatitis and hair loss etc.

Lead and Arsenic are major contaminant in our environment and it is present almost everywhere. In this study we have selected lead/Arsenic based cosmetics such as suruma and sindoor which are commonly used in day today life. Lead is a neurotoxin that is known to accumulate over time. It can cause neuronal damage and developmental delays even at extremely low concentrations. It can also cause learning, language and behavioural problems. ${ }^{2,3}$

The present study is designed to look for the students awareness about harmful effect of cosmetics and qualitative analysis of heavy metals from different types of cosmetics used on regular basis by students.

\section{METHODS}

This study was conducted in Yenepoya University, Mangalore, Karnataka from February 2014 to November 2014.

For the qualitative/quantitative estimation to detect heavy metals such as arsenic and lead in cosmetics, a questionnaire form was framed out in English with seven multiple choice questions and one descriptive type question. The forms were distributed to 150 students (of both the genders) of Yenepoya University.

A survey was conducted to find out the popular cosmetic brand used by the students of Yenepoya University and depending on the popularity 20 different cosmetics were purchased from local market. These cosmetics were categorized under Eye, Lips, Scalp and Hair, Nail and Skin. It was a single blind study and samples were coded (Table 1).

Preliminary analysis was carried out in Department of Forensic Medicine and Toxicology Research lab, Yenepoya University, Mangalore, Karnataka. by Copper wire method and Dithizone method after that the samples were digested using conc. $\mathrm{HNO}_{3}$. The coded samples were sent to IIT Bombay for ICP-AES analysis.

\section{RESULTS}

The use of cosmetics by humans started from an ancient time. They have been used by many different cultures for various reasons such as traditional, artistic or aesthetic purposes. $^{4}$

Table 1: Coding of samples for preliminary analysis.

\begin{tabular}{|ll|}
\hline Sample No. & Name of the Products \\
\hline Sample 1 & Skin foundation \\
\hline Sample 2 & Cream bleach \\
\hline Sample 3 & Powder \\
\hline Sample 4 & Skin whitening cream \\
\hline Sample 5 & Hair color \\
\hline Sample 6 & Facial Foundation \\
\hline Sample 7 & whitening cream \\
\hline Sample 8 & baby powder \\
\hline Sample 9 & Sindoor \\
\hline Sample 10 & Hair gel \\
\hline Sample 11 & Surma \\
\hline Sample 12 & Surma \\
\hline Sample 13 & Lip balm \\
\hline Sample 14 & Kajal \\
\hline Sample 15 & Nail Polish \\
\hline Sample 16 & Nail Polish \\
\hline Sample 17 & Hair removing cream \\
\hline Sample 18 & Hair removing cream \\
\hline Sample 19 & Cleansing milk \\
\hline Sample 20 & Powder \\
\hline
\end{tabular}

Questionnaire analysis of the boys and girls with respect to use of cosmetics (Figure 1) showed that the use of cosmetics for scalp and hair was more in boys when compared to the girls whereas the cosmetics for the eyes were used more by the girls.

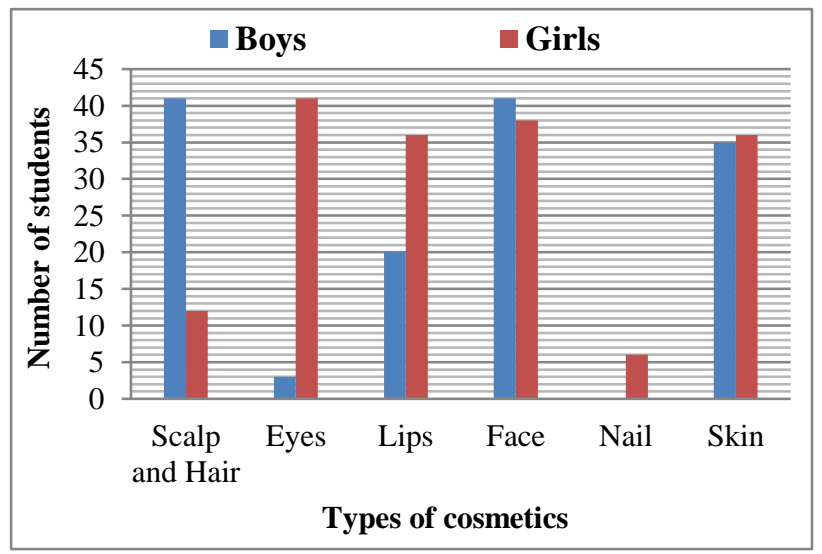

Figure 1: Types of cosmetics used by the students of Yenepoya University.

Questionnaire analysis also showed that adverse effect of cosmetics (Figure 2) were more in girls when compared to boys.

\section{Preliminary analysis of cosmetics}

All the reagents used in the study were of analytical grade and were purchased from Durga Laboratory Mangalore. 


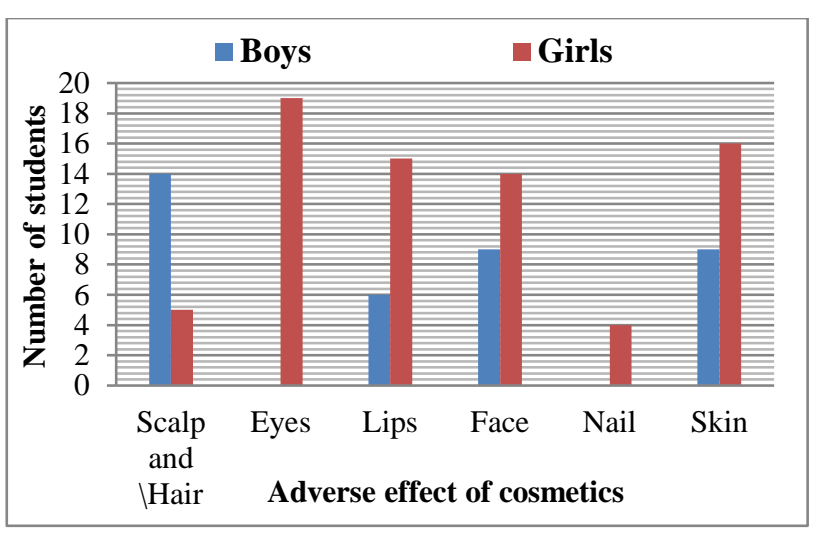

Figure 2: Adverse effect of cosmetics.

Preliminary analysis was done by Copper wire and Dithizone methods and some of the samples have given positive results. Results are given in Table 2 and Table 3.

Table 2: Qualitative test for Arsenic (copper wire method).

\begin{tabular}{|ll|}
\hline Sample No. & Presence / Absence Arsenic \\
\hline 1 & - \\
\hline 2 & - \\
\hline 3 & - \\
\hline 4 & - \\
\hline 5 & + \\
\hline 6 & - \\
\hline 7 & - \\
\hline 8 & - \\
\hline 9 & ++ \\
\hline 10 & - \\
\hline 11 & + \\
\hline 12 & + \\
\hline 13 & ++ \\
\hline 14 & - \\
\hline 15 & - \\
\hline 16 & - \\
\hline 17 & - \\
\hline 18 & - \\
\hline 19 & - \\
\hline 20 & - \\
\hline$-=$ Absent & \\
$+=$ Present in small quantity \\
$++=$ Present in moderate quantity \\
$+++=$ Present in large quantity \\
\hline
\end{tabular}

For the confirmation of the confirmation of results, the samples were sent to IIT Mumbai through courier for ICP-AES analysis and it was found that some of the products have shown variable levels of heavy metals such as lead and arsenic. Results are given in Table 4.

The levels of lead and arsenic found in twenty different cosmetics selected for the studies are given in Table 4. It was found that some of the products have shown variable levels of these metals, some being within the permissible limits and some well out of the safe range.
Table 3: Qualitative test for Lead (Dithizone method).

\begin{tabular}{|ll|}
\hline Sample No. & Presence / Absence Lead \\
\hline 1 & - \\
\hline 2 & - \\
\hline 3 & - \\
\hline 4 & - \\
\hline 5 & - \\
\hline 6 & - \\
\hline 7 & - \\
\hline 8 & + \\
\hline 9 & - \\
\hline 10 & + \\
\hline 11 & + \\
\hline 12 & + \\
\hline 13 & + \\
\hline 14 & + \\
\hline 15 & + \\
\hline 16 & - \\
\hline 17 & - \\
\hline 18 & - \\
\hline 19 & - \\
\hline 20 & \\
\hline- Absent & \\
\hline$=$ Present & \\
\hline
\end{tabular}

Table 4: ICP-AES analysis of cosmetic products.

\begin{tabular}{|lll|}
\hline Name of the products & \multicolumn{2}{l|}{$\begin{array}{l}\text { Concentration of the } \\
\text { heavy metal }\end{array}$} \\
\hline Lead & Arsenic \\
\hline Skin foundation & 0.49 & 0.40 \\
\hline Cream bleach & ND & ND \\
\hline Powder & ND & ND \\
\hline Skin whitening cream & 0.74 & 0.39 \\
\hline Hair color & 0.93 & 0.31 \\
\hline Facial Foundation & ND & ND \\
\hline Whitening cream & ND & ND \\
\hline Powder & ND & ND \\
\hline Sindoor & 644.86 & 6.40 \\
\hline Hair gel & ND & ND \\
\hline Surma & 1.23 & 3.18 \\
\hline Surma & 1437.50 & 3.48 \\
\hline Lip balm & 456.69 & 19.55 \\
\hline Kajal & 137.54 & ND \\
\hline Nail Polish & 2.96 & ND \\
\hline Nail Polish & 12.39 & ND \\
\hline Hair removing cream & ND & ND \\
\hline Hair removing cream & 0.061 & ND \\
\hline Cleansing milk & 0.70 & ND \\
\hline Powder & ND & ND \\
\hline
\end{tabular}

\section{DISCUSSION}

The usage of cosmetics for skin comprised mainly of creams, oils, and lotions which were found to be used equally by the both genders. Previous study by Kisan Shivaji Rao on consumer buying behaviour of cosmetic 
products, it was observed that even though the cosmetic market is dominated by female consumers, male consumers are coming at par with them. ${ }^{5}$

Increasing incidence of cosmetic usage of male consumer is another fact that observed in this study which is in correlation with the study done by Sangeetha et al. ${ }^{6}$

When asked about the signs and symptoms associated with cosmetic usage, $14 \%$ of the boys and 5\% girls agreed that they had adverse effects while using cosmetics for scalp and hair respectively. $19 \%$ of the girls had adverse effect while using cosmetics for eye, $15 \%$ of the girls had adverse effect while using low quality lip products. The adverse effect of using facial products was found in $14 \%$ of girls while $4 \%$ of girls had allergy with nail polish. Allergic response by the use of skin products was observed in $16 \%$ of girls. The percentage of the adverse effect seen in boys with regards to lip products, facial creams and skin products was found to be $6 \%, 9 \%$, and $9 \%$ respectively. Allergic reaction was manifested in the form of urticaria, pimples, darkening of skin, hair fall, skin irritation and loss of normal texture of hair etc.

Cristaudo et al, performed a cross-sectional study among 82 non-Italian women visiting an outpatient facility in Rome and Italy. It has been found that one fourth of these women were aware of potential risks and presence of high concentration of heavy metals $(\mathrm{Cr}, \mathrm{Ni}$ and $\mathrm{Pb})$ in these products. More than half of the women in the clinical evaluation had irritant contact dermatitis. ${ }^{7}$

It was found that the adverse reaction could be due to ingredients, contaminants, chemicals, heavy metals in the cosmetics, which is similar to the study done by Wioletta et al. ${ }^{8}$

Highest concentration of lead was found in Surma (1437.50 ppm) followed by Sindoor (644.86 ppm), Lip balm (456.69 ppm), Kajal (137.54 ppm), Nail polish (12.39 ppm). Normal safety range of lead is $20 \mathrm{ppm}$. Arsenic was detected in a number of cosmetics like skin foundation, skin whitening cream, hair colour, Sindoor, lip balm and Surma. Highest concentration of arsenic was found in Lip balm (19.55 ppm) followed by Sindoor (6.40 ppm), Surma (3.48 ppm). Normal safety range of arsenic is $3 \mathrm{ppm}$.

A study done by Sa Liu in a convenience sample of 32 lip products used by young women in Oakland and California found that most of the tested lip products contained high concentration of titanium, aluminium, manganese, cadmium and chromium, but in this study the lip products contained arsenic and lead which were above the safety limit. ${ }^{9}$ Chauhan et al, studied toxic metals in lipstick sold in Delhi region by using Flame Emission Spectrophotometer and found that most of the lipsticks contained chromium, lead, arsenic, cobalt, cadmium and nickel in variable amounts. ${ }^{10}$ Ramakant Sahu et al, studied heavy metals in different brands of cosmetic products such as lipsticks, lip-balm and anti-ageing creams, fairness cream and intake of heavy metals by body and found that all these products contain either chromium, nickel, lead, cadmium or mercury. ${ }^{11}$

Capelli et al, studied the toxic metals in face-powders and revealed the presence of all the considered metals in the range $0.06-8.0 \mu \mathrm{g} / \mathrm{g} .{ }^{12}$ Another study done by Nnorom et al, in the facial cosmetics found that the levels of chromium, iron and zinc were much higher in the samples but in this study lead and arsenic were not detected in facial cosmetics. ${ }^{13}$

Olabanji IQ and Oluyemi EA studied the concentrations of six heavy metals, Lead $(\mathrm{Pb})$, Nickel (Ni), $\mathrm{C}$ admium (Cd), Manganese (Mn), Chromium (Cr) and Arsenic (As) in most popular brand of ten lipsticks and ten nail polishes sold at cosmetic store in Ile-Ife market Nigeria by using Atomic Absorption Spectrophotometer (AAS). The results showed lead content in the nail polishes was in the range of $<0.00-42.14 \mathrm{ppm}^{.14}$ The lead values were found to be $12.39 \mathrm{ppm}$ and arsenic was not detected.

Jasmine et al, studied the levels of metals in nine samples of common hair-dye powders available in markets of Delhi, Lead and copper were detected in different concentrations in all the studied samples whereas in this study it was found that hair colouring agents contains both arsenic and lead and only lead was detected in one of the hair removing agent but all the values were within the safety range. ${ }^{15}$

This study has shown that cosmetics for eye and scalp contained potentially harmful heavy metals such as arsenic and lead. Surma contains highest concentration of lead (1437.50 ppm) and next is Sindoor (644.86 ppm) both these cosmetics products also contain Arsenic (Sindoor $6.40 \mathrm{ppm}$, Surma $3.48 \mathrm{ppm}$ ). Kajal contains 137.54 ppm of lead. Eeva-liisa Saino analyzed metals (lead, cobalt, nickel, chromium) and arsenic in eye shadows in 88 colours of 25 brands and 49 products. 66 out of $88(75 \%)$ of the colours contained more than 5 ppm of at least one of the elements, and all 49 products contained more than $1 \mathrm{ppm}$ of at least one of the elements. ${ }^{16}$ Study done by A.D Hardy et al, on comparison of eye cosmetics used in United Arab Emirates using 23 samples showed that all of these products contained lead. ${ }^{17}$ A comparative study of heavy metal content in cosmetic products of different countries marketed in Khyber Pakunkhwa, Pakistan by Hussain ullah et al. Also revealed that highest concentration of lead was found in Surma. ${ }^{18}$

\section{CONCLUSION}

Heavy metals are one of the common contaminant in our environment. Apart from this a number of cosmetic products also contain the same. Chronic use of this kind of cosmetic products will increase the level of trace metal in our body. 


\section{ACKNOWLEDGMENTS}

Authors would like to thanks Dr. Muhammed Rashid CS, Dr. Ramshid Abdul Aziz, P.G. Teaching and nonteaching staffs of Department of Forensic Medicine and Toxicology, Yenepoya Medical College, Mangalore, India for helping them in the preparation of the respective article.

\section{Funding: No funding sources}

Conflict of interest: None declared

Ethical approval: The study was approved by the Institutional Ethics Committee of Yenepoya University

\section{REFERENCES}

1. Barczac C. Hazards of cosmetics. (Place unknown): AEHA Quarterly summer;Environmental health association of Novascotia; 1995. (updated June 22 1995; cited 2014 Oct.). Available from: http://www.environmentalhealth.ca/summer95cosmet ic.html.

2. Vij K. Text book of Forensic Medicine and Toxicology, Principles and Practice. $5^{\text {th }}$ Ed. Elseiver, India; 2011.

3. Pillay VV. Modern Medical Toxicology. $4^{\text {th }}$ Ed. Jeypee Publisher, New Delhi; 2013.

4. Davis LC. African American women's use of cosmetics products in relation to their attitudes and self-identity (graduate thesis). Iowa State University, Ames, Iowa, US: 2013.

5. Rao KS. A study on consumer buying behavior of cosmetic products in Kolhapur. Reviews of literature. 2014;1(10).

6. Gupta S, Arora S. Factors influencing the Consumption Pattern of Male Cosmetics in Delhi by an Urban Male. IOSR $\mathbf{J}$ of Business and Management. 2013;15(2):29-36.

7. Cristaudo A, D'Ilio S, Gallinella B, Mosca A. Use of Potentially Harmful Skin-Lightening Products among Immigrant Women in Rome,Italy: A Pilot Study. Dermatology. 2013;226:200-6.

8. Wioletta A, Sobczak Z, Adamczuk P, Wróblewska P, Zwoliński J. Allergy to selected cosmetic ingredients. Postep Derm Alergol. 2013;XXX(5):307-10.
9. Liu S, Hammond SK, Cheatham AR. Concentrations and Potential Health Risks of Metals in Lip Products. Environ Health Perspect. 2013;121(6).

10. Chauhan SB, Chandak A, Agrawal SS. Evaluation of Heavy Metals contamination in Marketed Lipsticks. Intrntl J Advanced Research. 2014;2(4):257-62.

11. Sahu R, Saxena P, Johnson S. Heavy Metals in Cosmetics (project report). Centre for Science and Environment, Tughlakabad Institutional Area, New Delhi; 2014.

12. Capelli C, Foppiano D, Venturelli G, Carlini E. Determination of Arsenic, Cadmium, Cobalt, Chromium, Nickel, and Lead in Cosmetic FacePowders: Optimization of Extraction and Validation. Taylor and Francis. 2014;47(7):1201-9.

13. Nnorom IC, Igwe JC, Nnorom CG Oji. Trace metal contents of facial (make-up) cosmetics commonly used in Nigeria. African $J$ Biotechnology. 2005;4(10).

14. Ouremi OI, Ayodele OE. Lipsticks and Nail Polishes: Potential Sources of Heavy Metal in Human Body. Intnl $\mathrm{J}$ of Pharmaceutical Research and Allied Sciences. 2014;3(4):45-51.

15. Jasmine NR, Sharma P, Saroj NL. Determination of the Toxic Lead and Copper Levels in Cosmetic HairDye Powders by Flame Atomic Absorption Spectrophotometry. Medico-Legal Update. 2014;14(1):35-40.

16. Eeva-LiisaSainio EL, Jolanki R, Hakala E, Kanerva L. Metals and arsenic in eye shadows. Contact Dermatitis. Jan 2000;42(1):5-10.

17. Hardy AD, Sutherland HH, Vaishnav R. A study of composition of some eye cosmetics (Kohls) used in the United Arab Emirates. J Ethnopharmacol. 2002;80:137-45.

18. Ullah H. Comparative study of heavy metal content in cosmetic products of different countries marked in Khyber pakhtunkhwa, Pakistan. Arabian J Chemistry; 2013.

Cite this article as: Saidalavi R, Hashim A, Kumar KB, Pramod KL, Adake P. Analysis of lead and arsenic in cosmetics and assessment of students awareness about cosmetic toxicity. Int $\mathrm{J}$ Basic Clin Pharmacol 2017;6:1426-30. 